\title{
Reflexiones en torno a la consideración pedagógica de la educación 'formal', 'no formal' e 'informal'
}

\author{
JOSÉ ANTONIO JORDAN \\ Universidad Autónoma de Barcelona
}

\begin{abstract}
SUMMARY.-In this article, the author pretends -as already insinuates the proper title- to reflect upon the next points:

a) the mutual relation between 'formal', 'no-formal' and 'informal' education;

b) to question the proper concept of 'informal education';

c) to realize some epistemological considerations about the repercussions that the amplification of this object of knowledge can have on pedagogy, in its quality of science with a minimal proper entity;

It's on these two last aspects of this article, that really falls the principal weigth or effort of this study.
\end{abstract}

Este artículo es una reelaboración de la comunicación presentada en el $X$ Seminario Interuniversitario de Teoría de la Educación, en Llanes (Oviedo), en diciembre de 1991. El objeto de las reflexiones que siguen conviene ubicarlo dentro de un marco relativamente concreto, a saber, la ponencia, ciertamente brillante, del profesor Jaime Trilla: «La educación no formal. Definición, conceptos básicos y ámbitos de aplicación» ${ }^{1}$. Los aspectos que tematizo alrededor de dicha ponencia están descritos arriba, en el Sumario: a) interrelación entre las tres categorías de educación; b) la escurridiza conceptualización de la educación 'informal'; c) la repercusión epistemológica que puede tener para la Pedagogía la legítima ampliación de su objeto de estudio.

Comenzaré por el primer punto. En éste y otros lugares, el profesor Trilla señala que actualmente resulta insostenible identificar simplistamente 'educación' y 'escolarización'. En una visión prospectiva, incluso, no es aventurado esperar que el acento pedagógico tienda a situarse proporcionalmente más en el ámbito 'no-formal', dados los límites con que se topa la institución escolar para satisfacer las crecientes necesidades y demandas educativas. Los esfuerzos de la escuela por responder a las crecientes necesidades de aprendizaje en un tipo de sociedades progresivamente más complejas y concienciadas (e.g., alargando los años de escolaridad, incorporando en sus currícula acumulativas funciones, etc.) no logran $-\mathrm{y}$ cada vez menos- su propósito de forma mínimamente fructuosa. Como se expresa Ph. Coombs (1985): «Muchos países desarrollados se están convenciendo de que las tradicionales escuelas primarias no son los únicos,

1. Actualmente publicada en Sarramona, J. (1992): La educación no formal, Ceac, Barcelona, pp. 9-50. 
ni siquiera los mejores, medios para proporcionar a los niños y jóvenes las competencias y conocimientos básicos que necesitarán en la vida». De ahí, prosigue este autor, se derivará la urgencia de planteamientos más globales, comprehensivos, en relación con la satisfacción de las necesidades educativas de una comunidad (p. 1950). Estos vínculos entre las modalidades 'formal' y 'no formal', especialmente, tendrían su razón de ser pedagógica en una extensión cualitativa del concepto de educación (integral, permanente...) y en la mayor funcionalidad/flexibilidad que se precisa en no pocos ámbitos con similares objetivos educativos (e.g., en relación a grupos culturalmente minoritarios).

No se trata de analizar aquí el alcance y los límites de la escuela. En esta línea ya se han realizado interesantes estudios (Husen, 1986 y 1988; Trilla, 1985 a y b). Con todo, no está de más subrayar que la institución escolar puede seguir prestando una serie de servicios de difícil sustitución precisamente en sociedades y culturas de gran complejidad, como son las nuestras. Pocos dudarán, en este sentido, que puede ejercer un papel esencial en la transmisión sistemática de competencias básicas para «iniciar a los niños en una forma valiosa de vida», parafraseando la terminología de R. Peters (1982); competencias que, ciertamente, habrá que determinar cuidadosamente a través de rigurosos criterios pedagógicos (y sociológicos), así como también de condicionamientos pragmáticos (e.g., no engrosando de forma poco realista el currículum escolar con cada nueva exigencia educativa que aparece en el horizonte social). Sin entrar en pormenores, parece razonable que si el objetivo global de la escolarización obligatoria (supongamos al final de nuestra Educación Secundaria) es el de lograr sujetos básicamente 'educados', en cuanto capaces de 'iniciarse' en la vida sociocultural de la comunidad con un mínimo de garantías de éxito, los planteamientos curriculares escolares deberían hacer hincapié en conocimientos y destrezas de notable virtualidad funcional/transferencial, así como en actitudes especialmente positivas respecto al deseo de seguir aprendiendo.

Como también se ha subrayado abundantemente (Husen, 1978; Scribner y Cole, 1982), la institución escolar, en virtud de estar educando normalmente a través de unos formatos pedagógicos descontextualizados, cultiva primordialmente unas competencias (e.g., lenguaje 'elaborado', razonamiento abstracto...) a expensas de otras (e.g., capacidad de iniciativa, participación social, aprendizajes vitales...). Como indica el mismo T. Husen (1978: 26), la escuela suele modelar a los jóvenes en una existencia «rica en información, pero pobre en acción». La primacía del 'saber' sobre el 'saber hacer', ciertamente, es una característica usual de las influencias escolares; como también lo es, al menos en nuestras sociedades occidentales, la 'competitividad' sobre la 'cooperación' (a pesar de que ésta sea propuesta explícitamente a través de metas y métodos específicos, pero -al fin y el cabo- de forma normalmente sobreañadida).

Las consideraciones anteriores pretenden únicamente recordar que la institución escolar -en cuanto prototipo de la 'educación formal'- puede ser especialmente idónea para desarrollar cierto tipo de competencias, a la vez que notoriamente indigente para cultivar -en un grado mínimamente aceptable- otras (e.g.: del campo sociomoral, artístico, profesional... y del ocio). Todo ello no debería, sin embargo, llevar a la fácil conclusión de que la escuela debe renunciar a la educación 'sistemática' de éstas u otras dimensiones de la personalidad o, simplemente, que debe hacerlo de forma secundaria. Lo que cabe deducir, a mi juicio, es, más bien, que ha llegado el momento de empezar a pensar pedagógicamente en términos o expresiones como la propuesta por L. Cremin: «ecología de la educación» (cfr. Husen, 1988: 274), en tanto que invita a postular una 
Teoría global de la Educación. En un plano más práctico, esta orientación más abierta y comprehensiva del universo educativo debería conducir a planificaciones y políticas más flexibles y coordinadas; esto es, a partir de cuidadosos análisis de necesidades educativas de una población de amplitudes relativamente concretas (e.g.: de municipios), así como de inventarios detallados de las 'redes de aprendizaje' de carácter 'no formal', deberían empezarse a realizar planificaciones coherentes en relación a ofertas pedagógicas de todo tipo, tanto para cubrir las difícilmente soslayables limitaciones del sistema escolar a la hora de ofrecer una educación -realmente- básica, como para satisfacer las necesidades, deseos, expectativas..., personales/grupales respecto a ámbitos de aprendizaje concretos (desde el tiempo disponible propio de la 'tercera edad'... hasta la atención debida al cultivo de aspectos culturales minoritarios).

Más énfasis pondré en la segunda y tercera cuestión propuestas como objetos de estas reflexiones. Empecemos tratando, por ejemplo, algunos puntos problemáticos del concepto de educación informal. Este tema ha sido, por supuesto, objeto de serios estudios, entre los que cabe destacar aquéllos del profesor Trilla (1986 y 1992). Debe admitirse, en este sentido, que buena parte de este concepto ya ha sido analizado por el mencionado autor, así como por otros especialistas en la materia (Touriñan, 1984; García Carrasco, 1988; Castillejo, 1987; Quintana, 1991;... por citar algunos de nuestro contexto próximo). Sin embargo, la problemática de este 'nuevo' concepto es tal que bien pudiera ser útil ensayar algunas consideraciones más en torno a ella. No en balde, el mismo profesor Trilla sugiere en su última aportación (1992: p. 27, nota 27) que resulta muy relevante profundizar en ciertas cuestiones epistemológicas de este ámbito; por ejemplo: ¿conviene «formalizar» siempre, o como ideal pedagógico, los procesos de la educación informal? Discurramos un poco por ésta y otras vías del problema.

En relación a la distinción entre educación 'formal'- 'no formal', parece que se encuentra un cierto consenso (Touriñan: 1984; Trilla: 1992); a saber; en ambos casos se trataría de instituciones, procesos, medios..., «específicamente diseñados en función de explícitos objetivos educativos». La línea divisoria entre ambas categorías sería relativamente débil: la inclusión (actual) o no dentro del sistema educativo reglado; un criterio, por tanto, externo, legal. Esto no implica negar otro tipo de diferencias importantes: en la metodología, en la planificación, en la temporalidad, etc. Se trata, más bien, de subrayar que epistemológicamente ambas modalidades son formales, en cuanto que sus «formas» educativas son patentes..., es decir, manifiestamente pedagógicas.

En cambio, cuando de lo que se trata es de trazar la frontera entre las dos categorías anteriores y la tercera (educación 'informal') el asunto se torna más delicado. Entre otras cosas, están en juego dos aspectos espinosos: a) la intencionalidad del agente (personal o no); y b) la cuestión de los efectos educativos. Es aquí cuando empiezan las discrepancias.

Aunque muchos autores han problematizado hasta qué punto los procesos que no proceden de una intención específica o directa de provocar cambios educativos en el educando por parte del agente pueden incluirse rigurosamente dentro del concepto de educación, no va a ser éste el aspecto en que me detendré aquí. El prof. Trilla (1986: 107-196) ha tratado este problema con extensión y finura. Su conclusión viene a ser que, si queremos evitar un concepto de educación excesivamente angosto, hemos de concentrar nuestros criterios pedagógicos al respecto en los «efectos educativos» y no tanto en el «agente» que los provoca. El adjetivo (informal) haría referencia al mencionado agen- 
te, mientras que el sustantivo (educación) se basaría en el efecto producido (Ib.: 204). Ahora bien, procediendo así, resulta evidente que el foco de atención se concentra en una perspectiva concreta de abordar el concepto de educación; concretamente, en cuanto realidad o resultado, es decir, in facto esse. Aceptemos, de momento, que puede pensarse en la posibilidad de ampliar el concepto de educación en esta dirección y que la Pedagogía, en consecuencia, gana terreno en su objeto de estudio. El verdadero nudo gordiano, a mi entender, estriba en el segundo polo de la cuestión: en la problematicidad que encierra el discernir si un determinado efecto, o cambio de estado en la personalidad de un sujeto, es educativo o no, independientemente de la intencionalidad del agente, de si el proceso configurador está incluido indeferenciadamente en otras formas de influencia distintas.

Las discrepancias en este punto son importantes. Algunos prefieren etiquetar esta ampliación conceptual con el término «configuración» humana (Castillejo, 1987: 67), pues la «educación» implica siempre relación a un 'pattern' valioso (al menos en unas coordenadas socioculturales concretas). Dicho de otro modo: si bien es innegable que múltiples agentes incontrolados impactan en la conformación de nuestra personalidad (como insiste la corriente psicológica del life-span), habrían de descartarse como modificaciones 'educativas' las que no se adecúan al patrón humano tomado como referencia valiosa. De forma convergente razona J. M. Touriñan (1984: 124): «El hecho de que en educación informal se hable de resultados educativos obtenidos en procesos en los que el comunicador no se propone educar no significa que cualquier resultado o influencia sea educación»; de ser así - prosigue- «deberíamos defender erróneamente (e.g.) que influir en una persona para que deje de hacer lo que tiene que hacer para educarse es educación». Por tanto, según este autor, un efecto dejaría también de ser 'educativo' en el mismo momento lógico que cortase su relación con lo valioso, con lo humanamente optimizador (cf. también Marín, 1981; Sarramona, 1990: 42...; entre otros).

Desde luego, no se puede ocultar que el 'metro' para enjuiciar qué efectos configurativos deben entenderse como educativos y cuáles no resulta extremamente problemático. Este metro valorativo implica, ciertamente, aceptar un concepto de educación mínimamente prescriptivo (e.g., el propuesto por R. Peters alrededor del apetecido 'hombre racional'; cf. Jordan, 1989); por otro lado, lo deseable como auténticamente educativo ha sido frecuentemente juzgado con referentes morales, de forma que muchos influjos-efectos han sido desestimados como genuinamente educativos porque quedaban por debajo de ese techo (e.g., aprender hábitos higiénicos elementales por simple exposición a modelos y/o enseñanza verbal); cuestión que ha suscitado la clásica polémica sobre la distinción «instrucción»-«educación». Posiblemente este tipo de condicionantes, entre otros, han podido invitar al profesor J. Trilla a decantarse -conducido por su investigación centrada en la educación informal- por un concepto de lo educativo de gran amplitud: «Seguramente -dice- los criterios a utilizar deberán ser entonces los psicológicos (...), alguna clase de configuración de la personalidad» (del educando) (1986: 191; subrayado mío); lo que no obsta -añadirá poco más adelante- que después, en el orden lógico, pueda distinguirse entre 'buena' y 'mala' educación (Ib., p. 195). En síntesis, según este autor, a la Pedagogía le es conveniente, al menos en un primer momento epistemológico, contar con un objeto (la educación) amplio, sin constricciones axiológicas, culturales, etc. que lo empobrezcan heurísticamente (ib., p. 194). 
La solución adoptada por el profesor Trilla es muy razonable. De hecho, no pocos autores de relieve han intentado sortear el peligroso escollo del «valor educativo» de los efectos configuradores y de los fines de la educación mediante posiciones paralelas. Así hay que entender la postura formalista de los pedagogos afiliados al racionalismo crítico (e.g., F. von Cube, 1981; Brezinka, 1979). No obstante, en este caso el motivo no parte tanto de la voluntad de hacer más extenso el objeto de la Pedagogía, cuanto de hacer posible para ésta -dentro de sus presupuestos epistemológicos- un objeto susceptible de riguroso estudio científico; es decir, completamente aséptico respecto a todo contenido axiológico.

Sin pretensión, por supuesto, de resolver un tema tan problemático, sí que debo expresar mis reservas en relación a un concepto de educación informal tan amplio y, posteriormente, de su posible trascendencia epistemológica para la Pedagogía. Razonaré mi postura, abordando sintéticamente los puntos siguientes:

a) Conveniencia de un concepto de educación informal pedagógicamente aceptable.

b) Posibilidad de aproximarse a criterios mínimos para determina la valiosidad educativa de los diversos efectos configuradores.

c) Inconvenientes que puede tener para la Pedagogía un objeto excesivamente amplio.

En el primer punto simplemente quisiera manifestar que, dejando al margen el componente 'intencionalidad', se puede defender un concepto de educación informal que amplíe al máximo el horizonte del actual universo educativo, sin abdicar de la única conditio sine qua non: la de que el 'efecto' producido por influjos desconocidos o difusos posea 'valor educativo'. Esto parece razonable si, al margen de la carga de arbitrariedad que pueda suponer una parcelación epistemológica, la Pedagogía ha de tener como objeto propio y específico procesos/efectos netamente educativos. De lo contrario, como intentaremos mostrar en el análisis de la cuestión (c), la confusión del objeto puede derivar en la difuminación epistemológica de la ciencia que nos ocupa, de la Pedagogía.

Evidentemente, para poder acceder a un concepto de educación informal parcialmente restringido, se deben encontrar unos principios mínimos para descartar -al menos- los efectos des-educativos. El mismo profesor Trilla (1986: 151-153) menciona la importancia de una tarea de esta índole:

«Obviamente, no cualquier cambio en el sujeto constituye educación o 'efecto educativo'. Precisamente, la determinación rigurosa de lo que deba ser considerado como tal es uno de los problemas importantes de la Teoría de la Educación (...). Interesa destacar la relevancia que, para algunos temas de la teoría educativa, tendría una teoría de los efectos educativos». Y poco después: «Es una tarea actualmente inaplazable de la teoría de la educación el acotar con el máximo rigor y mínima ambigüedad lo que haya de ser considerado como efecto educativo (...). Pero a pesar de la gran relevancia que concedemos a esta cuestión (...), no creemos que esté dentro de nuestras posibilidades el desarrollarla aquí. Es tarea para otro trabajo».

Tampoco es éste el momento, ni el espacio, para abordar esta fundamental temática con la rigurosidad y detenimiento que merece. Pero permítaseme, siquiera, hacer algunas sugerencias al respecto. Un criterio de referencia para juzgar la valiosidad educativa de un efecto o influencia podría girar en torno a la definición ya mencionada de R. Peters: «iniciación del sujeto en una forma valiosa de vida». Cierto que en sociedades complejas y pluralistas, este criterio puede desembocar en dilemas conflictivos en relación al tema 
que andamos analizando; pero también es verdad que se puede inventariar un extenso listado de rasgos de personalidad altamente consensuados por los educadores y pedagogos de este tipo de sociedades (e.g., creatividad, iniciativa, crítica, competencia multilingüe y multicultural, autonomía..., un mínimo de conocimientos científico/técnicos, etcétera). Otro criterio útil puede hacer centro, no sólo en las necesidades adaptativas que tienen los sujetos respecto a un determinado entorno socio-cultural, sino también en la necesidad de satisfacer -mediante la educación- exigencias bio-psico-sociales (por no andar más lejos) del hombre en cuanto tal. Las tesis relativistas (y en su extremo la postmodernista), ciertamente, no secundan esta línea: algo vale en un contexto/cultura/momento... concretos. Pero, afortunadamente no todos piensan igual (cfr. Revel, 1989; Finkielkraut, 1990;... Jordan, 1991). Como magistralmente lo han ilustrado R. A. Levine y M. I. White (1987: 184), a través de la metáfora del 'arbusto', aunque las metas vitales y la forma de perseguirlas son tan variadas como las diferentes culturas existentes, «esto no quiere decir que las aspiraciones humanas sean infinitamente diversas. Todo el mundo desea sobrevivir, desea la salud y el bienestar material (...), la seguridad, el respeto...». Más aún, el hecho de poder hablar, por encima de las peculiaridades culturales concretas, de productos filosóficos, artísticos, científicos, literarios... de rango universal implica pensar en la posibilidad de que cada cultura -además de producir mecanismos para funcionar en un contexto discreto- desvela al conjunto de la humanidad formas de satisfacer necesidades genéricamente humanas. En suma; sin necesidad de acudir inmediatamente al horizonte metafísico, cabe mostrar (si no demostrar) que «todas las culturas, o todo lo que se dice llamar cultura, no vale igual» (Jordan, 1992: cap. III). El tercer criterio, en fin, que se me ocurre para el problema de los valores educativos es de carácter netamente pedagógico, y merece un comentario especial.

En otro estudio (Jordan, 1990) ya he tematizado con cierto detenimiento una problemática íntimamente conexa a ésta: La complejidad epistemológica de los fines de la educación. No repetiré, pues, ahora los comentarios que allí hice. Considero, sin embargo, que puede ser útil recordar algunas consideraciones básicas. Una de ellas es la clara distinción que hace J. M. Touriñan (1989) entre 'fines educativos' y 'valores'; los primeros serían fruto de una elección con criterios pedagógicos de entre un cúmulo de valores (filosóficos, sociales..); el que la competitividad, e.g., sea un valor social evidente (aunque a veces encubierto) no implica, según este autor, que deba ser aceptado acríticamente por la Pedagogía. Todavía más; el valor 'espíritu crítico', educativamente positivo, puede no ser elegido como 'fin educativo', con criterios técnico-pedagógicos, para alumnos de edades muy tempranas. Ahora bien, resulta palmario que, previamente a la conveniencia técnica-circunstancial para aceptar un valor como fin educativo (e.g., el caso del 'sentido crítico' para edades tempranas), la Pedagogía ha tenido que discriminar qué valores son 'científico-técnicamente' educativos y cuáles no. Como apunta el profesor Touriñan, al igual que la Medicina juzga los valores médicos con el criterio del valor 'salud', la Pedagogía (científico-tecnológica) puede delimitar un conjunto de valores educativos en cuanto que facilitan directa o indirectamente 'educación'. Dicho de otro modo, la Pedagogía, en cuanto subsistema o ámbito de conocimiento epistemológicamente independiente -al menos, relativa y funcionalmente- podría dictaminar con criterios intrínsecos, admitidos consensuadamente por los pedagogos en cuanto tales, qué cambios en la personalidad del educando son educativamente valiosos. Por ejemplo, el aprendizaje 'informal' por parte del niño de conductas/actitudes de puro consumo a tra- 
vés del influjo televisivo sería, en la perspectiva anterior, deseducativo, no sólo por recurrir a criterios ideológico/morales, sino porque pedagógicamente dichos efectos configuradores riñen con funciones psicológicas aceptadas positivamente en el marco de la ciencia pedagógica (e.g., el sentido crítico, la autonomía e iniciativa, etc.). El tema es fecundo y -como ya ha apuntado el profesor Trilla- merecedor de iniciativas en la línea de desarrollar una Teoría Pedagógica (descriptiva y normativa) de los efectos educativos.

Había un tercer punto en relación a este tema que también queríamos comentar mínimamente. Me refiero a los inconvenientes que para la Pedagogía puede tener en el plano epistemológico la aceptación de un concepto de educación informal demasiado amplio. Como ha mostrado el profesor Trilla (1986: 77 ss.), la educación informal está íntimamente conectada -y frecuentemente diluida- en otros procesos cercanos: socialización (adaptación a la vida social de un grupo), enculturación (adquisición de la cultura de dicho grupo) y aprendizaje social (mecanismos psicológicos para adaptarse y aculturarse). Sin embargo, resulta de nuevo útil insistir que de no seleccionar los efectos configurativos valiosos educativamente como los únicos -y hay muchísimos- dignos de constituir el objeto directo de la Pedagogía en la extensión de su radio de acción, esta ciencia acaba diluyéndose epistemológicamente en otras próximas (e.g., la Sociología, la Antropología cultural, la Psicología Social, etc.). Este interés por acotar lo más definidamente posible nuestro espacio epistemológico radica en la necesidad de evitar todo asomo de «colonización disciplinar» de otras ciencias respecto a la nuestra (Vázquez, 1980: 52). Todo ello no quiere decir, evidentemente, que la Pedagogía no deba recoger todos los aportes de estas ciencias afines; todo lo contrario, le interesa muchísimo conocer las formas en que han sido socializados y enculturizados los niños objeto de educación (e.g., esto es fundamental a la hora de plantear estrategias didácticas diferenciadas para niños culturalmente minoritarios, con distintos 'estilos cognitivos', en el marco de una educación multicultural); pero, insisto, todo ello lo puede hacer indirectamente desde la perspectiva epistemológica. Por lo demás, resulta difícil admitir -a pesar del patente solapamiento- que cualquier proceso de socialización o enculturación produzca resultados educativos (e.g., el aprendizaje de actitudes individualistas a partir de multitud de estímulos de nuestra sociedad/cultura occidental), como para incluirse inmediatamente en el objeto central de la ciencia pedagógica.

En el propósito de esta contribución estaba también incluida la intención de reflexionar sobre la trascendencia que tiene para la Pedagogía el ampliar su objeto de estudio (a través de la educación 'no formal' y, en particular, de la 'informal'). La constricción del espacio, sin embargo, obligará a un tratamiento simplificado en exceso de esta cuestión. Indicamos, no obstante, algunos aspectos.

$1^{\circ}$ ) Parece cada vez más necesaria una Teoría de la Educación (Pedagogía) de carácter global (Scribner; Cole, 1982: 4; Husen, 1988: 274), llamada a describir y explicar las abundantes «formas educativas» actualmente ocultas en el seno de otras modalidades socioculturales de influencia, es decir, a conocer los mecanismos configurativos de rasgos de la personalidad educativamente positivos. Y esto puede aplicarse también respecto a los procesos 'informales' incrustrados difusamente en el curso de las mismas instituciones y actividades catalogadas de 'formales' y 'no formales'. Por ejemplo, respecto al complejo «currículum oculto» (Vázquez, 1985), a la virtualidad educadora de los «medios o climas» educativos (García Carrasco, 1988), a los metamensajes que provoca la personalidad del profesor según la teoría de los «modelos» (Aznar; Vega, 1986), 
etc. Es cierto que algunas Ciencias de la Educación (e.g., Sociología de la Educación, Historia de la Educación...) ya han comenzado a inscribir dentro de su objeto de estudio ámbitos de la educación no convencionales (no formal e informal) (Trilla, 1984), pero no es menos verdad también que queda todavía un largo camino en lo que se refiere a la tarea de enriquecer el cuerpo de la Pedagogía, en cuanto ciencia normativa con un corpus de conocimientos y estrategias de intervención procedentes de estas categorías no escolares. Convertir en «formales» (y no formales) la procesos informales, una vez explicados; instrumentalizarlos pedagógicamente mediante el pertinente control tecnológico-educativo, constituye uno de los más interesantes retos que tiene ante sí la Pedagogía actual (Trilla, 1986: 241). Baste como ejemplo las propuestas de aprendizaje 'natural' de la lectura, música... propuestos para el aprendizaje temprano de estas competencias por R. Cohen (1983).

$2^{\circ}$ ) Ahora bien, no cabe duda de que esa tarea inaplazable deviene, a la vez, una dinámica heurística nunca acabada; es decir, siempre quedará un poso de educación informal, algo de azaroso y descontrolado en la propia instrumentalización pedagógica 'formal'; acicate -ciertamente- de la continua investigación pedagógica (Trilla, 1981: 114).

$3^{\circ}$ ) Finalmente, otra cuestión digna de reposada reflexión es la de si esa reconversión de lo 'informal' en 'formal' es conveniente siempre y/o en todo ámbito educativo. Responder afirmativamente significa pedagogizar la vida entera del hombre, intentar no dejar ningún cabo suelto, procurar un progresivo 'control' del hombre y de su circunstancia en pro de metas - en principio- educativamente valiosas. Sin poder matizar aquí este interesante interrogante, si que se adivinan posturas -al menos parcialmente- divergentes. La concepción de las «ciencias de la educación... (como) sociotecnologías» (Bunge, 1985: 337) o de «la educación... (como) el vector más relevante del proceso 'configurativo' de la especie humana» (Castillejo, 1987: 36), por citar algunos ejemplos, irían en la dirección de una respuesta afirmativa a la cuestión planteada. Entre nosotros también, si no estoy equivocado en mis apreciaciones, el profesor J. García Carrasco (1983: 173) concede menos alcance -aún vinculado a la postura intervencionista- a la virtualidad pedagógica: «El pedagogo -comenta- no es un profesional que pretenda hacer al hombre (...), porque la vida es el todo, y no existe un punto de apoyo en que colocar la pértiga para moverla». Las citas y opiniones de un signo y otro se podrían multiplicar, pero no es este el momento de escudriñar en este problema esencial. $\mathrm{Me}$ conformaré con dejar abiertas algunas preguntas: ¿debe instrumentalizarse pedagógicamente todo tiempo de ocio; ...todos los momentos del día; ...todas las dimensiones humanas (morales, políticas...); ...todas las relaciones interpersonales...?

\section{BIBLIOGRAFÍA}

AzNAR, P.; VegA, F. (1986). «Tecnología educativa de los modelos»; en Revista Teoría de la Educación, $\mathrm{n}^{\circ} 1$, pp 169-181.

BrezinKa, W. (1979). La Scienza dell'Educazione, Armando Armando, Roma.

Bunge, M. (1985). Seudociencia e ideología, Alianza Universidad, Madrid.

Castillejo, J. L. (1987). Pedagogía Tecnológica, Ceac, Barcelona. 
COHEN, R. (1983). En defensa del aprendizaje precoz, Planeta, Barcelona.

Coombs, Ph. (1985). «Formal and Nonformal Education. Strategies for the Future»; en Husen, T.; Potlewaite, S. N. (eds.): International Encyclopedia of Education, Pergamon Press, Oxford and New York, pp. 1949-1951.

Cube, F. von (1981). La ciencia de la educación, Ceac, Barcelona.

FinkielKraut, A. (1990). La derrota del pensamiento, Anagrama, Barcelona.

García Carrasco, J. (1983). La ciencia de la Educación. Pedagogos ¿para qué?, Santillana, Madrid.

García CARRASCO, J. (1988). «Agentes de la educación formal, no formal e informal», en Symposium Internacional de Filosofia de l'Educació (ponencias), U.A.B., Bellaterra, pp. 127-155.

HUSEN, T. (1978). La sociedad educativa, Anaya, Madrid.

HUSEN, T. (1986). La escuela a debate, Narcea, Madrid.

HusEn, T. (1988). Nuevo análisis de la sociedad del aprendizaje, Paidos/Mec, Madrid.

JORDAN J. A. (1989). «El constructo 'hombre educado'. Un análisis epistemológico/antropológico»; Comunicación presentada en el II Congreso Nacional de Teoría de la Educación, febrero, Torremolinos, $12 \mathrm{p}$.

JORDAN J. A. (1990). «Problematicidad epistemológica de los fines de la educación», Educar, 14/15, pp. 9-33.

JORDAN J. A. (1991). «¿Valen igual todas las culturas? Una cuestión previa a la Educación Multicultural»; Comunicación presentada en el III Congreso Nacional de Teoría de la Educación: Educación Multicultural y Europa, Madrid, 15 p.

JoRDAN J. A. (1992). L’Educació Multicultural, Ceac, Barcelona.

LEVINE, R. A.; WHITE, M. I. (1987). El hecho humano. Las bases culturales del desarrollo educativo, Visor/Mec, Madrid.

MARín, R. (1983). «La educación como optimización del hombre»; en Varios: Teoría de la Educación (I) (El problema de la educación), Ed. Límites, pp. 107-123.

Peters, R. (1982). «La educación y el hombre educado»; en Dearden, R. F. et al.: Educación y desarrollo de la razón, Narcea, Madrid, pp. 21-34.

QUiNTANA, J. M Ma (1991). «La educación más allá de la escuela»; en Varios: Iniciativas sociales en educación informal, Rialp, Madrid, pp. 15-61.

ReVel, J. F. (1989). El conocimiento inútil, Planeta, Barcelona.

SARRAmona, J. (1990). Tecnología Educativa, Ceac, Barcelona.

SCRIBNER, S.; COLE, M. (1982). «Consecuencias cognitivas de la educación formal e informal», Infancia y aprendizaje, $\mathrm{n}^{\circ} 17$, pp. 3-18.

TouRiÑAN, J. M. (1984). «Análisis teórico del carácter 'formal', 'no formal' e 'informal' de la educación»; en Papers d'Educació. Conceptos y Propuestas (I), Nau Llibres, Valencia, pp. 109-133.

ToURIÑAN, J. M. (1989). «Las finalidades de la educación: Análisis teórico»; en Esteve, J. M. (Ed.): Objetivos y contenidos de la educación para los años noventa, Universidad de Málaga, pp. 15-36. 
TriLla J. (1981). «Un límite a la afirmación de lo educativo como sistémico», Educació i Cultura, $\mathrm{n}^{\mathbf{0}}$ 2, pp. 107-114.

TRILla J. (1984). «Sobre l'aplicació dels conceptes d'educació informal i educació no formal a la Historia de l'educació»; Actas de les Sisenes Jornades d'Historia de l'Educació als Països Catalans, 8-9-10 març., Escola Universitària de Magisteri, Lleida, pp. 134-147.

TRILla J. (1985 a). Ensayos sobre la escuela, Laertes, Barcelona.

TRILla J. (1985 b). La educación fuera de la escuela, Planeta, Barcelona.

TRILLA J. (1986). La educación Informal, PPU, Barcelona.

TRILlA J. (1992). «El sistema de educación no formal: Definición, conceptos básicos y ámbitos de aplicación»; en Sarramona, J. (ed.): La educación no formal, Ceac, Barcelona, pp. 9-50.

VÁZQUEZ, G. (1980). «Unidad, autonomía y normatividad en la investigación pedagógica»; en Actas del VII Congreso Nacional de Pedagogía, CSIC, Madrid, vol. II, pp. 39-61.

VÁZQUEZ, G. (1985). «Currículum oculto y manifiesto»; en Varios: Condicionamientos sociopolíticos de la educación, Ceac, Barcelona, pp. 167-187. 